\title{
Dificuldades e concepções de licenciandos em Matemática a respeito dos conceitos de dependência e independência linear ${ }^{1}$
}

\author{
Mariany Layne de Souza \\ Universidade Estadual de Londrina - UEL \\ marianylayne@gmail.com
}

\section{Angela Marta Pereira das Dores Savioli ${ }^{2}$}

Universidade Estadual de Londrina - UEL

angelamarta@uel.br

\begin{abstract}
Resumo
Este artigo apresenta resultados de uma pesquisa que objetivou investigar dificuldades e concepções, segundo o aporte teórico APOS (action-process-object-schema), referentes aos conceitos de dependência e independência linear de licenciandos em Matemática. Para tanto, analisaram-se os registros escritos de cinco questões que foram aplicadas em uma turma do segundo ano da Licenciatura em Matemática de uma universidade do norte do Paraná. Conclui-se, com relação às dificuldades, que grande parte dos participantes da pesquisa apresentaram alguma dificuldade no que diz respeito: à linguagem empregada na Álgebra Linear; ao entendimento dos conceitos de dependência e independência linear; à identificação dos conjuntos linearmente dependentes e independentes e ao reconhecimento da representação gráfica de vetores linearmente dependentes. E conclui-se, com relação às concepções, de acordo com o aposte teórico APOS, que houve a manifestação da concepção ação e da concepção processo, sendo que a concepção ação foi a mais evidenciada nos licenciandos.
\end{abstract}

Palavras-chave: Educação Matemática. Dificuldades. Aporte teórico APOS. Álgebra Linear. Dependência e independência linear.

\section{Difficulties and conception of undergraduates in Mathematics about of Linear Dependence and Independence Concepts}

\begin{abstract}
This article presents results of a research that aimed to investigate difficulties and conception, according to the APOS (action-process-object-schema) theoretical framework related to linear dependence and linear independence on concepts of undergraduates in Mathematics. Therefore, the written records analyzed five questions that have been applied to a class of second year of the Degree in Mathematics from a north university of Paraná. It is concluded in relation to the difficulties that

\footnotetext{
${ }^{1}$ Este artigo é resultado da pesquisa de mestrado da primeira autora (SOUZA, 2016), que contou com o apoio financeiro da Coordenação de Aperfeiçoamento de Nível Superior (CAPES).

${ }^{2}$ A segunda autora contou com apoio da Fundação Araucária, via convênio 288/2012, protocolo.19179.
} 
the majority of survey participants had some difficulty with regard: the language used in linear algebra; the understanding of the concepts of linear dependence and independence; the identification of linearly dependent and independent sets and recognition of the graphical representation of linearly dependent vectors. And concludes with respect to conceptions, according to the APOS theoretical framework, there was the manifestation of the action design and design process, and design the action was most evident in the undergraduates.

Keywords: Mathematics Education. Difficulties. APOS theoretical framework. Álgebra Linear. Linear dependence and independence.

\section{Introdução}

A Álgebra Linear é um assunto importante não apenas à Matemática, mas também no que diz respeito à sua aplicação em diversas áreas do conhecimento, como, por exemplo, Física, Química, Economia, dentre outras (DORIER, 2000).

As preocupações com o ensino e a aprendizagem da disciplina de Álgebra Linear podem decorrer devido a essa gama de aplicações e também por ser evidente em pesquisas, como a de Celestino (2000), na qual essa disciplina está dentre as que mais causam reprovação, o que revela as dificuldades que os estudantes apresentam com sua aprendizagem.

Levando em consideração esse estado da situação, muitas pesquisas têm sido realizadas, como a de Stewart (2008), a de Karrer e Jahn (2009), dentre outras, com o intuito de compreender e encontrar novas alternativas para a sala de aula, a fim de auxiliar no ensino e na aprendizagem dos conceitos de Álgebra Linear. Alguns trabalhos, como o de Dorier e Sierpinska (2001), também evidenciam diferentes causas para as dificuldades dos estudantes, entre elas, o formalismo e a variedade de linguagens que são empregadas nos estudos dos conceitos.

Valendo-se dessas reflexões, desenvolvemos esta pesquisa, que teve por objetivo investigar dificuldades e concepções referentes aos conceitos de dependência e independência linear de licenciandos em Matemática, usando o aporte teórico APOS (ação - processo - objeto - esquema). Para tanto, as análises dos registros escritos dos participantes foram embasadas nas dificuldades que podem ser manifestadas, valendo-se de trabalhos como o de Dorier e Sierpinska (2001), e do APOS desenvolvido por Dubinsky e seus colaboradores (ASIALA, et al. 1996).

\section{Dificuldades em Álgebra Linear}

As questões relacionadas às dificuldades apresentadas por estudantes na disciplina de Álgebra Linear são objeto de estudo de autores como Dorier e Sierpinska (2001), Stewart (2008), dentre outros. 
Dorier e Sierpiska (2001) comentam que as dificuldades em Álgebra Linear podem ter dois tipos de origem: uma associada à dificuldade conceitual, que se relaciona à sua natureza, e outra, à dificuldade cognitiva, que se refere aos tipos de pensamento necessários para o seu conhecimento.

O primeiro tipo de origem, segundo os autores, pode estar relacionado com o fato de os estudantes não perceberem o caráter unificador, generalizador e simplificador em um primeiro curso de Álgebra Linear.

Outro aspecto abordado, a partir da dificuldade conceitual, diz respeito ao caráter formal da linguagem utilizada no estudo da Álgebra Linear. Essa dificuldade é associada ao fato de os alunos não compreenderem a especificidade do uso do formalismo, o que pode ocasionar o "obstáculo do formalismo" (DORIER; SIERPINSKA, 2001). Além disso, Dorier e Sierpinska (2001) comentam que podem ser empregadas diferentes linguagens no estudo da Álgebra Linear, sendo elas a geométrica, a algébrica e a abstrata. Os autores, valendo-se de Duval (1995), tratam também dos registros gráfico, tabular e simbólico, que podem ser utilizados, e das diferentes conversões de registro semiótico necessárias em algumas atividades.

Para tratar do segundo tipo de origem, a dificuldade cognitiva, Dorier e Sierpinska (2001) abordam a flexibilidade cognitiva, discutida por Alves Dias e Artigue (1995), mostrando que os alunos necessitam transitar entre as conversões de registro semiótico livremente, o que pode não acontecer por eles ainda não terem essa flexibilidade.

Além disso, Dorier e Sierpinska (2001) falam da dificuldade dos estudantes chegarem ao nível de pensamento trans-objetal, que corresponde à construção de estruturas, pelo fato de os mesmos não atribuírem significado para os símbolos e terminologias que utilizam na Álgebra Linear. Os autores comentam a respeito do pensamento prático em oposição ao teórico, em que os estudantes mostram uma tendência a basear sua compreensão em exemplos, ao invés de se basear na definição do objeto matemático.

Por fim, os autores tratam dos modos de pensamento em Álgebra Linear, sendo eles o aritmético-analítico e o estrutural-analítico. As dificuldades desses modos de pensamento estão relacionadas, algumas vezes, às atividades que são apresentadas; assim, retorna-se à questão da flexibilidade cognitiva que é necessária ao estudante.

Outra pesquisa que apresenta algumas razões para as dificuldades dos estudantes em Álgebra Linear é a de Stewart (2008). Dentre as razões levantadas pela autora estão a natureza abstrata da Álgebra Linear (que de certo modo foi brevemente abordada nesta seção), a falta de problemas adequados e o papel das definições no conhecimento matemático. 
Com relação à falta de problemas adequados, Stewart (2008) comenta que é difícil encontrar problemas para cada novo conceito e que, assim, os estudantes acabam se queixando pelo fato de acharem o curso de Álgebra Linear muito abstrato.

Ao tratar do papel que as definições possuem em relação às dificuldades da disciplina de Álgebra Linear, a pesquisadora em questão argumenta, com base no conceito definição e conceito imagem (TALL; VINNER, 1981), que as interpretações dos estudantes a respeito das definições podem causar dificuldades na aprendizagem, o que, em certa medida, evidencia que elas podem salvar o estudante de muitas armadilhas, no que se refere a aplicações posteriores, e, ainda, evitar diversos equívocos que surgem caso a imagem do objeto matemático não seja baseada no conceito definição.

Há ainda outro trabalho, o de Andreoli (2009), que faz uma análise dos obstáculos encontrados pelos alunos ao estudarem os conceitos de dependência e independência linear. A autora elenca alguns desses obstáculos, como, por exemplo, o "encerramento no uso das definições de LD [linearmente dependente] e LI [linearmente independente] como única ferramenta possível" e "a nãoinclusão da solução trivial entre as infinitas soluções de um sistema homogêneo indeterminado" (ANDREOLI, 2009, p. 145). Esses obstáculos podem ser encarados como dificuldades dos estudantes ao estudarem Álgebra Linear.

Mediante o que foi apresentado nesta seção, podemos observar que as dificuldades com a Álgebra Linear podem possuir um caráter subjetivo e outro relacionado com a sua natureza. Dessa forma, apresentamos um quadro-síntese (Quadro 1), com o intuito de organizar e resumir as dificuldades abordadas anteriormente.

Quadro 1 - Síntese das dificuldades

\begin{tabular}{|c|c|}
\hline \multirow{2}{*}{$\begin{array}{l}\text { Dificuldade com a } \\
\text { natureza da Álgebra Linear }\end{array}$} & Caráter formal da linguagem empregada na Álgebra Linear. \\
\hline & $\begin{array}{l}\text { Diferentes linguagens empregadas nos conceitos (algébrica, geométrica, } \\
\text { abstrata). }\end{array}$ \\
\hline \multirow{4}{*}{ Dificuldade subjetiva } & $\begin{array}{l}\text { Flexibilidade cognitiva para transitar entre as diferentes formas de registros } \\
\text { (gráfico, tabular, simbólico). }\end{array}$ \\
\hline & Não atribuição de significado para os símbolos e as terminologias utilizadas. \\
\hline & Interpretação das definições dos conceitos. \\
\hline & Estratégias para identificar vetores LI e LD. \\
\hline
\end{tabular}

Fonte: Elaborado pelas autoras

\section{Aporte teórico APOS}

O aporte teórico APOS (action-process-object-squema) foi desenvolvido pelo grupo de pesquisadores denominado Research in Undergraduate Mathematics Education Community (RUMEC), que buscava compreender a abstração reflexionante (réfléchissante) introduzida por 
Piaget (1972), uma vez que em seus estudos foi dito que tal abstração, em sua forma mais avançada, possibilitaria a conversão dos processos em objetos matemáticos. Essa abstração é considerada como sendo a construção mental dos objetos (DUBINSKY, 2002).

Com relação à construção do conhecimento matemático, de acordo com Asiala et al. (1996), ela passa pelas etapas ação, processo, objeto e esquema.

A concepção ação corresponde a uma transformação que o indivíduo realiza sobre um objeto matemático. Essa transformação acontece devido à reação dada às indicações que fornecem o passo a passo a respeito de como realizar uma operação. Tais indicações são obtidas, às vezes, quando o indivíduo recorre à sua memória; com isso, pode ser que ele não tenha controle consciente do que está fazendo. Um exemplo da concepção ação seria aplicar a definição para identificar conjuntos linearmente dependentes (LD) e independentes (LI) (KÚ, TRIGUEIROS, OKTAÇ, 2008).

A concepção processo é uma construção interna que ocorre quando uma ação é repetida várias vezes e o indivíduo reflete a respeito dela, a interiorizando em processo. No processo o indivíduo passa a realizar uma transformação consciente, conseguindo fazê-la sem ter estímulos externos e podendo descrever o passo a passo sem explicitá-lo. Tem-se, como exemplo da concepção processo, o fato de o sujeito perceber que para se ter vetores $\mathrm{LD}$, um vetor deve ser escrito como combinação linear dos outros (STEWART, 2008).

A concepção objeto se refere a uma reflexão que o indivíduo realiza no processo, ou seja, o indivíduo vê o processo como um todo e percebe que pode fazer transformações conscientes nele, por meio de ações e/ou processos. Com isso o processo é encapsulado em objeto. Um exemplo da concepção objeto se dá quando um indivíduo consegue perceber os conceitos em sua totalidade, podendo visualizar tais características em representações gráficas, ou até mesmo sendo capaz de associar a questão de dimensão de um espaço vetorial para identificar se um conjunto pode ser LI ou LD.

O esquema é formado por uma coleção de ações, processos e objetos que são organizados de forma estruturada na mente do indivíduo. Para exemplificar a concepção esquema podemos pensar em um estudante aplicando os conceitos de dependência e independência linear em situações diversas e em outros espaços vetoriais diferentes do $R^{n}$.

\section{Procedimentos metodológicos}

A coleta de dados apresentada neste artigo ocorreu por meio dos registros escritos, obtidos por um instrumento com cinco questões, aplicado em dois dias a dezessete estudantes do segundo ano do curso de Licenciatura em Matemática. Esses registros foram analisados de forma minuciosa 
tomando como base o referencial adotado. É interessante dizer que a pesquisa em questão possui caráter qualitativo.

A análise foi feita em dois momentos. No primeiro investigamos as dificuldades, utilizando para tanto a análise textual discursiva que possui como elementos principais: a desmontagem dos textos; o estabelecimento de relações e captando o novo emergente (MORAES; GALIAZZI, 2007). Já no segundo momento investigamos as concepções, à luz do aporte teórico APOS, realizando inferências com base nos registros escritos dos participantes da pesquisa.

A delimitação do corpus foi realizada utilizando dois critérios: ter respondido totalmente ou parcialmente o instrumento de coleta de dados, a fim de ter material suficiente para realizar inferências sobre as dificuldades e as concepções, e ter participado dos dois dias de aplicação. Dessa forma, onze licenciandos fazem parte do corpus de análise. Para identificá-los e manter o anonimato adotamos um código empregando a letra $\mathrm{E}$ seguida de um número para diferenciá-los (E1, E2, ...).

\section{Um olhar para as dificuldades}

Nesta seção direcionamos nosso olhar para as dificuldades, usando para analisá-las a análise textual discursiva. Para tanto, elencamos uma única categoria denominada dificuldades. Essa categoria apresentou quatro subcategorias de análise: linguagem; entendimento dos conceitos de dependência e independência linear; identificação de um conjunto LI e LD, e reconhecimento da representação gráfica. A partir disso, buscamos identificar e discutir as dificuldades dos licenciandos com relação aos conceitos de vetores LI e LD.

Para as três primeiras subcategorias, mencionadas anteriormente, usamos as seguintes unidades de análise prévias: adequada, parcialmente adequada e inadequadas. Para a quarta subcategoria elaboramos duas unidades de análise prévias: reconheceu e não reconheceu.

$\mathrm{Na}$ subcategoria linguagem, que pretende identificar como os estudantes valem-se da linguagem utilizada na AL para responder as questões do instrumento de coleta de dados, foram efetivadas duas unidades de análise: adequada e inadequada (Quadro 2). Para esta subcategoria e para as demais apresentamos apenas alguns registros escritos como exemplos, devido ao tamanho do artigo. 
Quadro 2 - Subcategoria linguagem e unidades de análise efetivadas

Unidade de análise: adequada

Será verificado se os estudantes utilizam de forma adequada a linguagem empregada em Álgebra Linear.

1) 0 que significa dizer que vetores são linearmente dependentes ou linearmente independentes?

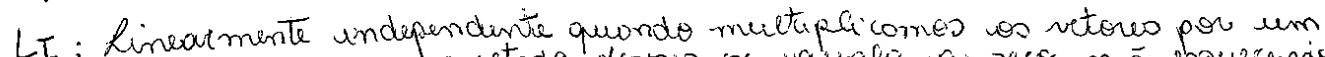
escalar qualquer es o resuetodo desses se uqualia a zeco. nos ebquerenas

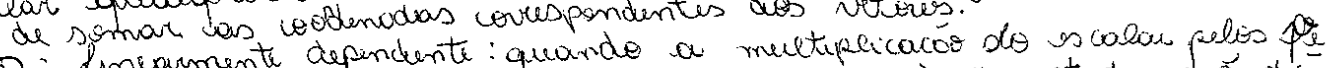

LD: Lnvarmente depindente quando gero ou seja seus resucto dos beo dé

Tores resultar en

$(\mathrm{E} 3)^{3}$

3) Um aluno da disciplina de Álgebra Linear fez o seguinte comentário: "Os elementos do conjunto $A=\{(1,2,1),(1,-1,0),(2,3,4)\}$, contido em $R^{3}$, são linearmente independentes, porque podem ser escritos como uma combinação linear". Você concorda' com essa afirmação? Justifique.

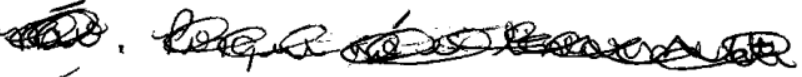

nais, por que so quo nolo is linearmentu

colepholente que pode ser escrito em

uma combinaçâs lines

Unidade de análise: inadequada

Será verificado se os estudantes utilizam de forma inadequada a linguagem empregada em Álgebra Linear.

1) $O$ que significa dizer que vetores são linearmente dependentes ou linearmente

independentes? Ds vetoress linearmente depen-

dentes são vetores, que podem, vere

seja, iräo depender dos nimeros reais

que multiplicam essess vetores. As vetores

linearmente indeperdentes não podem ser escrita

- como combinaça linear. .

1) $O$ que significa dizer que vetores são linearmente dependentes ou finearmente independentes?

Linearmente dependente í quando wos valores dos resultodos sōo diferentes.

Linearmente indzpendente i quando es volores dos resultadoz

sōo iguais a zero.

Fonte: Elaborado pelas autoras

\footnotetext{
${ }^{3}$ Transcrição do registro escrito de E3 - LI: Linearmente independente quando multiplicamos os vetores por um escalar qualquer e o resultado desses se iguala a zero não esquecendo de somar as coordenadas correspondentes dos vetores. LD: Linearmente dependente: quando a multiplicação do escalar pelos vetores resulta em diferente de zero ou seja seis resultados são diferentes de zero.
} 
Na subcategoria entendimento dos conceitos de dependência e independência linear, que visa verificar como os estudantes interpretam e entendem tais conceitos, foram efetivadas as três unidades de análise prévias (Quadro 3).

Quadro 3 - Subcategoria entendimento dos conceitos de dependência e independência linear e unidades de análise efetivadas

Unidade de análise: adequado

Será verificado se a interpretação e o entendimento a respeito dos conceitos são adequados.

1) $O$ que significa dizer que vetores são linearmente dependentes ou linearmente independentes?

Quer dige que seus coeficientes linares dependem on nä́o dos valous dos attos eveficients para subermos seur valires.

3) Um aluno da disciplina de Álgebra Linear fez o seguinte comentário: "Os elementos do conjunto $A=\{(1,2,1),(1,-1,0),(2,3,4)\}$, contido em $R^{3}$, são linearmente independentes, porque podem ser escritos como uma combinação linear". Você concorda com essa afirmação? Justifique. Too concondo, poi esta afinmaigo refere se a veloves Linearmenle dependentes.

Unidade de análise: parcialmente adequado

Será verificado se a interpretação e o entendimento a respeito dos conceitos são parcialmente adequados.

1) O que significa dizer que vetores são linearmente dependentes ou linearmente independentes?

Vitores linwares dependentes os retores que depende de outro para so obter 0 resulto do i independento é ocontrario

3) Um aluno da disciplina de Álgebra Linear fez o słguninte comentário: "Os elementos do conjunto $A=\{(1,2,1),(1,-1,0),(2,3,4)\}$, contido em $R^{3}$, são linearmente independentes, porque podem ser escritos como uma combinação linear". Você concorda com essa afirmação? Justifique.

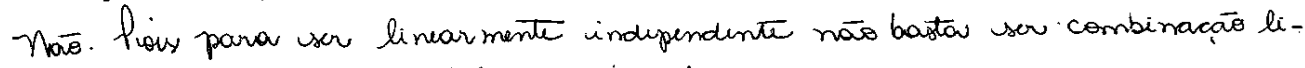
near í necessário que os vetores se iqualem a zero.

\footnotetext{
${ }^{4}$ Transcrição do registro escrito de E5 - Quer dizer que seus coeficientes lineares dependem ou não dos valores dos outros coeficientes para sabermos seus valores.

${ }^{5}$ Transcrição registro escrito de E6 - Não concordo, pois esta afirmação refere-se a vetores linearmente dependentes.

${ }^{6}$ Transcrição registro escrito de E1 - Não. Pois para ser linearmente independente não basta ser combinação linear é necessário que os vetores se igualem a zero.
} 
Unidade de análise: inadequado

Será verificado se a interpretação e o entendimento a respeito dos conceitos são inadequados.

3) Um aluno da disciplina de Álgebra Linear fez o seguinte comentário: "Os elementos do conjunto $A=\{(1,2,1),(1,-1,0),(2,3,4)\}$, contido em $R^{3}$, são linearmente independentes, porque podem ser escritos como uma combinação linear". Você concorda com essa afirmação? Justifique.

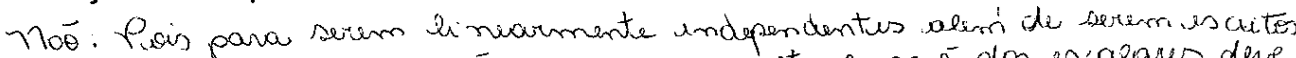
como uma combenacae ennear, a multupli acas dos escalares deve

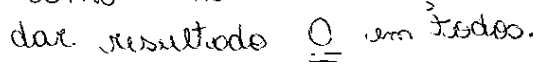

3) Um aluno da disciplina de Álgebra Linear fez o seguinte comentário: “Os elementos do conjunto $A=\{(1,2,1),(1,-1,0),(2,3,4)\}$, contido em $R^{3}$, são linearmente independentes, porque podem ser escritos como uma combinação linear". Você concorda com essa afirmação? Justifique.

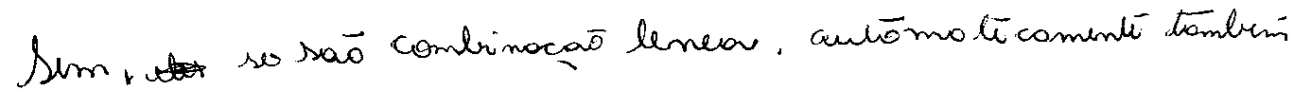

Jao di

Fonte: Elaborado pelas autoras

Na subcategoria identificação de um conjunto LI e LD, que visa observar como os estudantes identificam e mostram tais conceitos, foram efetivadas as três unidades de análise e foi necessário elaborar uma unidade emergente com relação à não-identificação (Quadro 4).

\section{Quadro 4 - Subcategoria identificação de um conjunto LI e LD e unidades de análise efetivadas}

Unidade de análise: adequada

Será apurado se os estudantes identificam de forma adequada quando um conjunto é LI ou LD.

2) İentifique dentre os conjuntos $A=\{(1,2),(2,4),(3,6)\}$ e $B=$

$\{(1,1,1),(3,-1,2),(0,-4,-1)\}$ quais possuem vetores linearmente dependentes. Por quê? (Adaptado de Oliveira, 2005, p. 111).

$a(1,2)+b(2,4)+c(3,6)=0$

$(a, 2 a)+(a b, 4 b)+(3 c, 6 c)=0$

$(a+2 b+3 c, 2 a+4 b+6 c)=0$

$a+a b+3 c=0$

$\left\{\begin{array}{l}a+2 b+3 c=0 \\ 2 a+4 b+6 c=0\end{array}\right.$
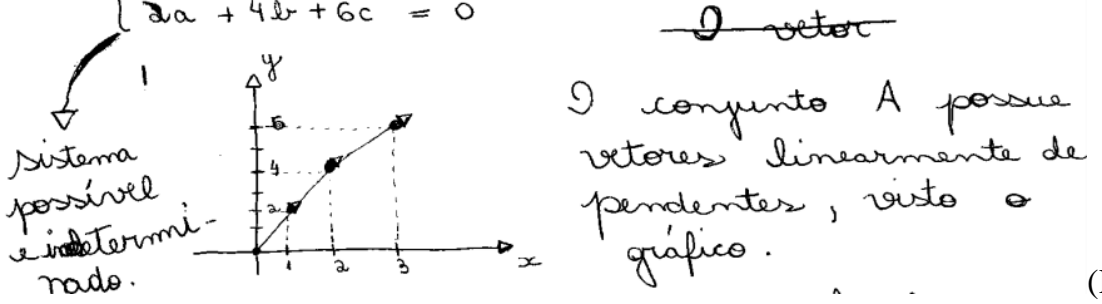

(E2)

\footnotetext{
${ }^{7}$ Transcrição registro escrito de E3 - Não. Pois para serem linearmente independentes além de serem escritos como uma combinação linear, a multiplicação dos escalares dever dar resultado 0 em todos.
} 


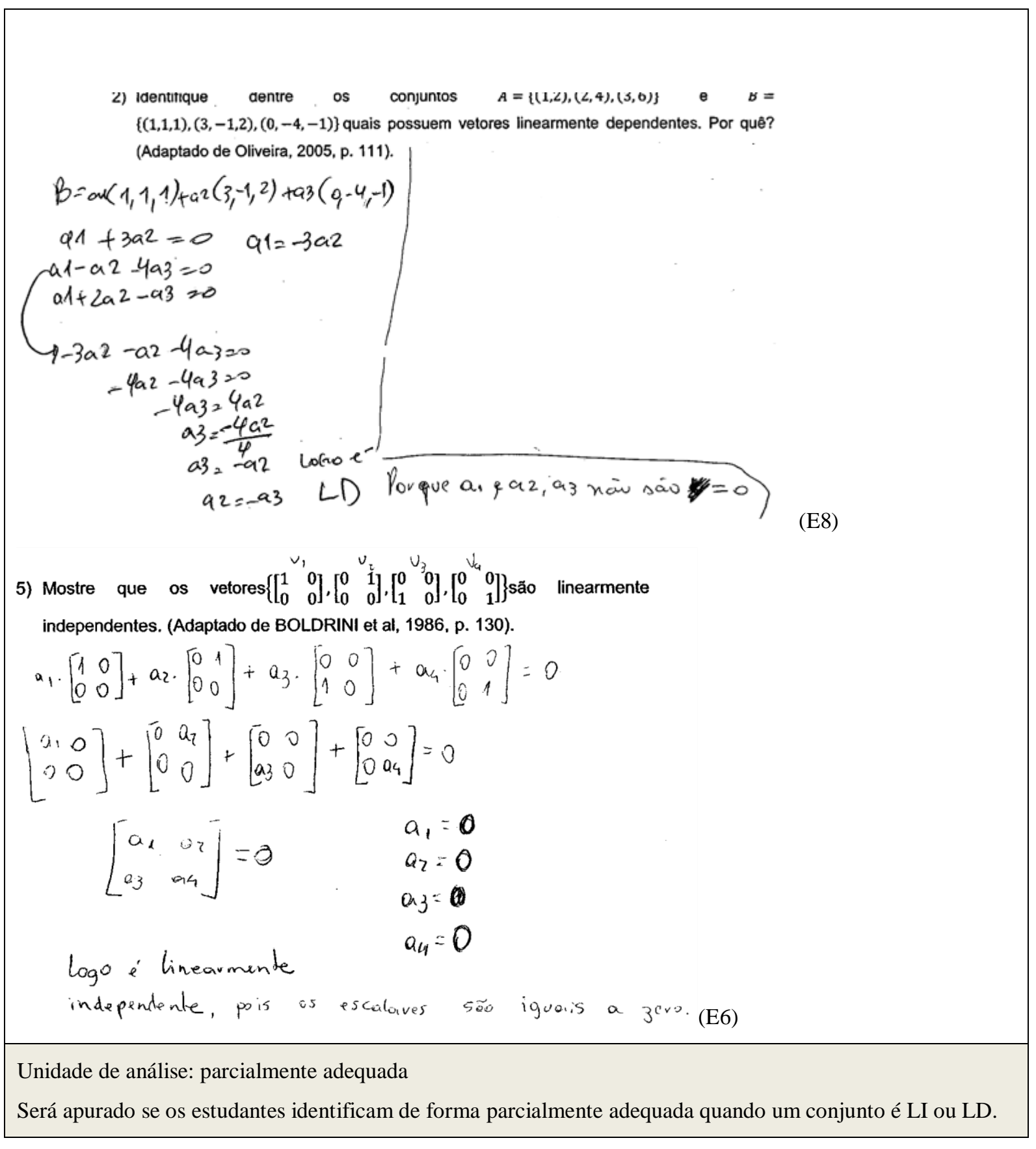




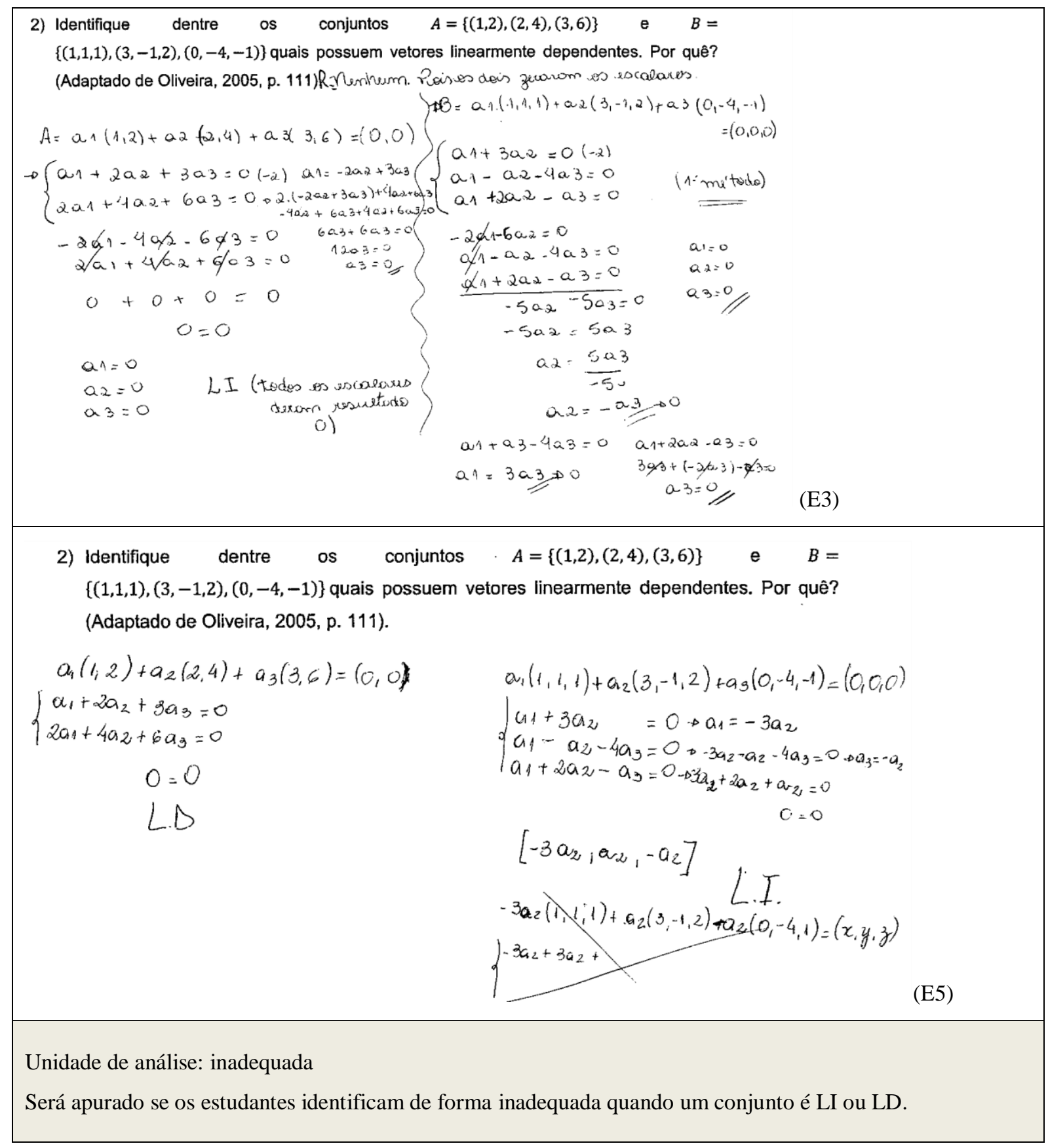


2) Identifique dentre os conjuntos $A=\{(1,2),(2,4),(3,6)\}$ e $B=$ $\{(1,1,1),(3,-1,2),(0,-4,-1)\}$ quais possuem vetores linearmente dependentes. Por quê? (Adaptado de Oliveira, 2005, p. 111).

a. $\{(1,2),(2,4),(3,6)\}$

$B\{(1,1,1),(3,-1,2),(0,-4,-1)\}$

$A=(1+2+3,2+4+6)$

$(6,12)$

(18)

Is dois persuem vtous linearmente dependentes. Perque a sama dos

$B=(1+3+0,1-1-4,1+2-1)$ seurs votous i diferente de

$(4,-5,2)$ zero.

$$
(4-5+2)
$$

$(-1)$

5) Mostre que os vetores $\left\{\left[\begin{array}{ll}1 & 0 \\ 0 & 0\end{array}\right],\left[\begin{array}{ll}0 & 1 \\ 0 & 0\end{array}\right],\left[\begin{array}{ll}0 & 0 \\ 1 & 0\end{array}\right],\left[\begin{array}{ll}0 & 0 \\ 0 & 1\end{array}\right]\right\}$ são linearmente

independentes. (Adaptado de BOLDRINI et al, 1986, p. 130).

$\left[\begin{array}{ll}1 & 0 \\ 0 & 0\end{array}\right]+\left[\begin{array}{ll}0 & 1 \\ 0 & 0\end{array}\right]+\left[\begin{array}{ll}0 & 0 \\ 1 & 0\end{array}\right]+\left[\begin{array}{ll}0 & 0 \\ 0 & 1\end{array}\right]$

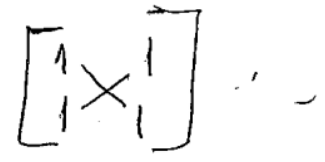

$1-1=0$

(E10)

Unidade de análise: não identificou

Será apurado se os estudantes não conseguiram identificar quando um conjunto é LI ou LD.

2) Identifique dentre os conjuntos $A=\{(1,2),(2,4),(3,6)\}$ e $B=$ $\{(1,1,1),(3,-1,2),(0,-4,-1)\}$ quais possuem vetores linearmente dependentes. Por quê? (Adaptado de Oliveira, 2005, p. 111).

noò lembro como se resolve.

2) Identifique dentre os conjuntos $A=\{(1,2),(2,4),(3,6)\}$ e $B=$

$\{(1,1,1),(3,-1,2),(0,-4,-1)\}$ quais possuem vetores linearmente dependentes. Por quê? (Adaptado de Oliveira, 2005, p. 111).

não lumbro

Fonte: Elaborado pelas autoras 
Para a última subcategoria, reconhecimento da representação gráfica, que busca verificar se os estudantes conseguem reconhecer a representação gráfica de vetores LI, foram efetivadas as duas unidades de análise (Quadro 5).

Quadro 5 - Subcategoria reconhecimento da representação gráfica e unidades de análise efetivadas

Unidade de análise: reconheceu

Será verificado se os estudantes conseguiram reconhecer a representação gráfica

4) Os vetores $v_{1}$ e $v_{2}$ representados na figura abaixo são linearmente independentes? Justifique.

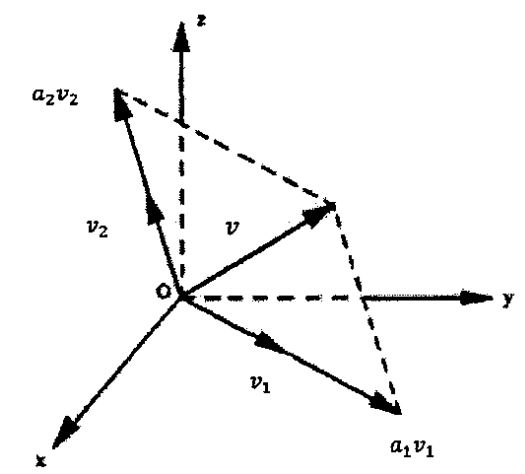

Fonte: STEINBRUCH; WINTERLE, 2009, p. 43.

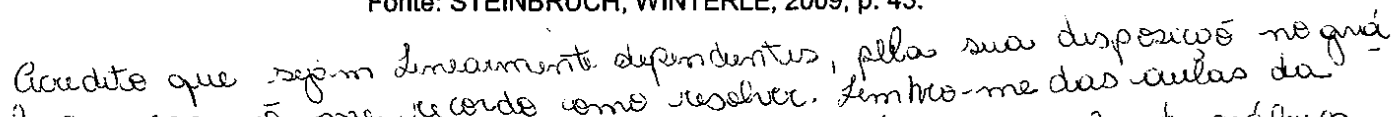

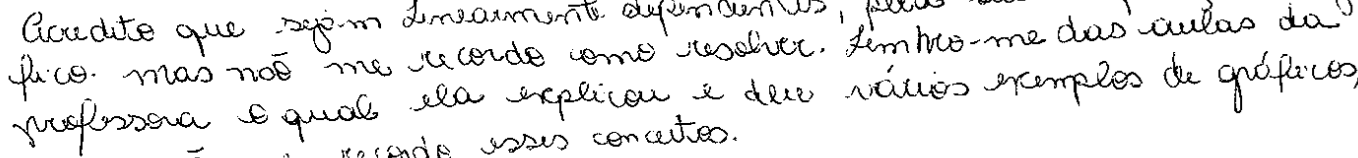
mas nos uni recorde usses concetion.

Unidade de análise: não reconheceu

Será verificado se os estudantes não conseguiram reconhecer a representação gráfica 
4) Os vetores $v_{1}$ e $v_{2}$ representados na figura abaixo são linearmente independentes? Justifique.

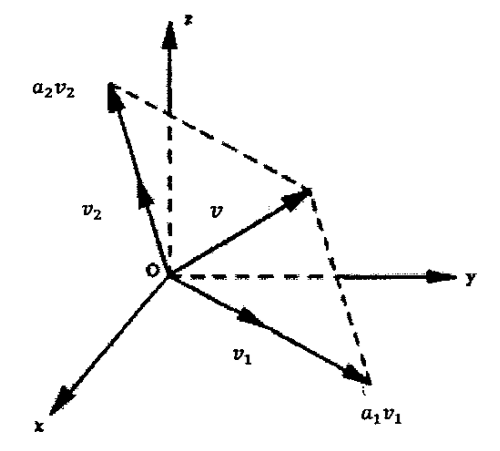

Fonte: STEINBRUCH; WINTERLE, 2009, p. 43.

Sâo, pois de acoudo com o gráfico les gram o rto $\theta$.

4) Os vetores $v_{1}$ e $v_{2}$ representados na figura abaixo são linearmente independentes? Justifique.

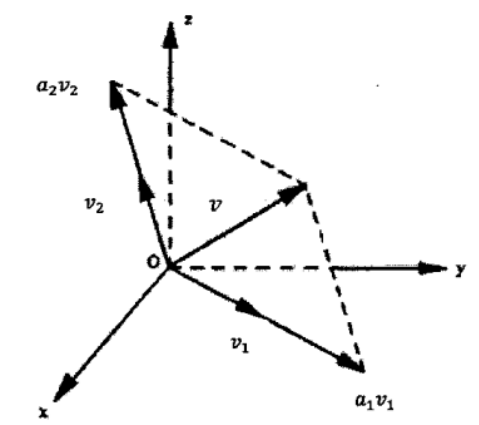

Fonte: STEINBRUCH; WINTERLE, 2009, p. 43.

nä́sei identificer

Fonte: Elaborado pelas autoras

\section{Síntese das dificuldades: o metatexto}

A categoria dificuldades contemplou quatro subcategorias: linguagem, entendimento dos conceitos de dependência e independência linear, identificação de um conjunto de vetores LI e LD, e reconhecimento da representação gráfica.

Observamos com a subcategoria linguagem que a maioria dos licenciandos (E3, E4, E8, E9, E10, E11) não mostrou dificuldade com a linguagem empregada na Álgebra Linear. Os demais a apresentaram, por associar, por exemplo, a palavra escalares a valores dos resultados (E7). Com essa subcategoria, notamos que essa dificuldade pode ocorrer devido à Álgebra Linear valer-se de um caráter formal, e que, algumas vezes, os estudantes não entendem a necessidade da especificidade de 
tal uso, o que pode caracterizar o "obstáculo do formalismo", como mostrado por Dorier e Sierpinska (2001).

$\mathrm{Na}$ subcategoria entendimento dos conceitos de dependência e independência linear, percebemos que a maioria dos estudantes apresenta um entendimento parcialmente adequado (E1, E4, E5, E7, E8, E11), quando se trata de uma das características de vetores LD, pois, por exemplo, associaram a combinação linear ao vetor nulo, e não aos vetores dados na terceira questão (E1, E8, E11). Vemos com essa subcategoria que o entendimento que os licenciandos apresentam a respeito de tais conceitos pode fazer com que caiam em certas armadilhas (STEWART, 2008), por não associarem algumas características desses conceitos.

Na subcategoria identificação de um conjunto LI e LD, vários licenciandos (E1, E3, E4, E5, E6, E7, E8, E9) revelaram uma identificação adequada ao mostrar que um conjunto era LI; porém, alguns tiveram certo problema com a notação por igualar uma combinação linear de matrizes a um número real (E3, E4, E5, E6, E7). Quanto à identificação de um conjunto de vetores LD, a maioria dos licenciandos a fizeram de forma parcialmente adequada (E3, E4, E5, E6, E8, E10), uma vez que, por exemplo, associaram a igualdade $0=0$ a vetores LI (E3, E8, E10). Percebemos, assim, que grande parte dos licenciandos valeu-se do "uso das definições de LD e LI como única ferramenta possível" (ANDREOLI, 2009, p. 145) para identificar e/ou mostrar se um conjunto é LI ou LD. Constatamos, ainda, que alguns equívocos quanto às estratégias utilizadas levaram à identificação parcialmente adequada e inadequada desses conceitos.

$\mathrm{Na}$ última subcategoria, reconhecimento da representação gráfica, parte dos estudantes não conseguiu atingir o objetivo da questão, algumas vezes por não se recordar (E8 e E11), outras por confundir as representações gráficas (E4, E5, E6, E9, E10). Isso nos leva a perceber a dificuldade dos licenciandos em identificar uma representação gráfica, às vezes, por não associarem as características de vetores LI e LD, o que pode estar relacionado ao entendimento de tais conceitos (STEWART, 2008). Tal situação pode ter relação também com a falta de "flexibilidade cognitiva" (ALVES DIAS; ARTIGUE, 1995) necessária para os estudantes transitarem entre os diferentes tipos de registros de representação semiótica.

Mediante o que foi exposto, pode-se constatar que grande parte dos licenciandos em Matemática, participantes desta pesquisa, apresentou alguma dificuldade, que pode estar atrelada tanto à natureza quanto ao caráter subjetivo da Álgebra Linear.

\section{Um olhar para as concepções}


Nesta seção direcionamos nosso olhar para as concepções dos licenciandos em Matemática. Desse modo, investigaremos cada concepção, segundo a APOS, comentando as resoluções feitas por três estudantes, com a intenção de exemplificá-las. No entanto, ao término desta seção apresentaremos a síntese com todos os estudantes.

\section{Concepção ação: licenciando E5}

O licenciando E5 resolveu todas as questões do instrumento aplicado. Ao dar suas respostas percebemos que tal estudante se vale, de certa forma, da definição, recorrendo à expressão que pode ser utilizada para identificar vetores LI ou LD, o que evidencia que E5 não interiorizou uma das características de vetores LD, ou seja, escrever um dos vetores de certo conjunto como combinação linear dos demais, e não reconhece uma representação gráfica de vetores LD. Além disso, notamos que E5 comete alguns equívocos como obter um sistema possível e indeterminado e dizer que o conjunto de vetores era LI igualando uma combinação linear de matrizes a um número real.

A partir das respostas dadas às questões, podemos inferir que E5 possui concepção ação, uma vez que busca identificar quais vetores são LD, vendo aqueles que podem ser escritos como combinação linear do vetor nulo. É possível perceber ainda que o estudante não interiorizou algumas ações, como, por exemplo, se há a possibilidade de se escrever um vetor como combinação linear de outros vetores, então, esses vetores são LD.

\section{Concepção processo: licenciando E2}

O licenciando E2 resolveu parcialmente as questões do instrumento aplicado. Ao dar suas respostas, observamos que o estudante se vale da definição, mas também das características de vetores LD e LI e reconhece a representação gráfica de vetores LD.

Por meio das respostas dadas por E2 às questões do instrumento, é possível perceber que tal estudante apresenta concepção processo, pois interiorizou alguns aspectos da dependência e independência linear, como a expressão que representa a combinação linear com o vetor nulo e a possibilidade de escrever vetores de um conjunto como combinação linear de outros ser uma característica de vetores LD.

\section{Indícios da concepção ação: licenciado E11}

O licenciando E11 resolveu parcialmente as questões do instrumento aplicado. Por meio de suas respostas notamos que o estudante recorre a procedimentos equivocados, como realizar adições entre os componentes dos vetores, calcular o determinante de cada componente do vetor, no caso do conjunto de matrizes $M(2,2)$. Esses equívocos podem ter sido cometidos possivelmente por E11 
associar alguns fragmentos de seu entendimento a respeito dos conceitos de LD e LI ou por associar alguns outros conceitos vistos na disciplina de Álgebra Linear.

Com base nas respostas dadas por E11 às questões do instrumento de coleta de dados, observamos que tal estudante apresenta alguns conceitos equivocados em relação à dependência e independência linear, evidenciando indícios de que ainda está construindo esses conceitos.

\section{Síntese das concepções, segundo a APOS, evidenciadas nos licenciandos}

Após olhar as resoluções dos estudantes e inferir suas concepções, à luz do aporte teórico APOS, apresentaremos um quadro síntese (Quadro 6) do que foi evidenciado nos licenciandos em Matemática.

Quadro 6 - Concepções evidenciadas nos licenciandos

\begin{tabular}{|c|c|}
\hline Concepções & Licenciandos \\
\hline Concepção ação & E1, E3, E5, E7, E8, E9 \\
\hline Concepção processo & E2, E6 \\
\hline Somente indícios da concepção ação & E4, E10, E11 \\
\hline
\end{tabular}

Fonte: Elaborado pelas autoras

Percebemos que, dos onze licenciandos investigados, seis apresentaram concepção ação dos conceitos de dependência e independência linear, o que revela que eles ainda possuem uma noção elementar de tais conceitos.

\section{Considerações finais}

Com o objetivo de investigar dificuldades e concepções referentes aos conceitos de dependência e independência linear de licenciandos em Matemática, analisamos os registros escritos de onze licenciandos.

Dessas análises identificamos dificuldades quanto à linguagem, ao entendimento dos conceitos de dependência e independência linear, à identificação de conjuntos LI e LD e ao reconhecimento da representação gráfica.

Observamos que nessas dificuldades houve problemas em relação ao formalismo que a Álgebra Linear exige. Alguns licenciandos recorriam a termos equivocados para justificar suas respostas, outros cometiam equívocos quanto a notação, dentre outros. Essa questão do formalismo é discutida por diversos pesquisadores, como Dorier e Sierpinska (2001), e vista como uma das causas de dificuldades dos estudantes. 
Em relação às concepções, identificamos que os licenciandos em Matemática apresentaram em sua maioria a concepção ação, o que leva à conclusão de que nesse primeiro curso de Álgebra Linear que participaram não construíram uma concepção esquema dos conceitos de dependência e independência linear, mostrando ainda uma noção elementar destes.

Além disso, podemos relacionar as dificuldades manifestadas pelos estudantes em relação às concepções apresentadas por eles, uma vez que muitos problemas relacionados ao entendimento e à notação contribuíram para inferirmos qual concepção os licenciandos apresentaram.

Vemos, com isso, que as dificuldades acabam se entrelaçando com as concepções que podem ser manifestadas pelos estudantes, segundo a APOS. Dessa forma, com intuito de possibilitar meios que amenizem as dificuldades e contribuam para que os estudantes construam seus conhecimentos dos objetos matemáticos em sua totalidade, é necessário que se levem em conta vários fatores, como o reconhecimento da natureza abstrata da Álgebra Linear, e se busque refletir a respeito da forma de ensinar tal disciplina, pensando nos diversos rumos que os estudantes podem tomar na construção de seus conhecimentos.

\section{Referências}

ANDREOLI, Daniela Inés. Análisis de los obstáculos en la construcción del concepto de Dependencia Lineal de vectores en alumnus de primer año de la universidad. 2009. $194 \mathrm{f}$. Dissertação (Mestrado en Ciencias en Matemática Educativa). Centro de Investigación y em Ciencia Aplicada y Tecnologia Avanzada, Instituto Politécnico Nacional, Distrito Federal México, 2009. Disponível em:

<http://www.matedu.cicata.ipn.mx/tesis/maestria/andreoli_2009.pdf>. Acesso em: 20 jan. 2015.

ALVES DIAS, Marlene. ARTIGUE, Michelle. Articulation Problems Between Different Systems of Representations in Linear Algebra. In: International Conference for the Psychology of Mathematics Education, 19., Pernambuco, 1995. Proceedings... São Paulo: Atual Editora, 1995. Volume 2. 290 p. $42-49$.

ASIALA, Mark, et al. A Framework for Research and Curriculum development in Undergraduate Mathematics Education. In: KAPUT, J., et al. (Eds.). Research in Collegiate Mathematics education, 2. Washington: American Mathematical Society, 1996, p. 1-32. Disponível em: <http://www.math.kent.edu/ edd/Framework.pdf>. Acesso em: 08 jul. 2014.

CELESTINO, Marcos R. Ensino-Aprendizagem da Álgebra Linear: as pesquisas brasileiras na década de 90. 2000. 114 f. Dissertação (Mestrado em Educação Matemática) - Pontifícia Universidade Católica de São Paulo, São Paulo, 2000.

DORIER, Jean-Luc. Use of history in a research work on the teaching of linear algebra. In: KATZ, V. (Ed.). Using history to teach mathematic - An international perspective. Washington D.C.: The Mathematical Association of America (Inc.), 2000, p. 99-110. Disponível em: <https://archiveouverte.unige.ch/unige:16850> Acesso: 05 jan. 2015.

DORIER, Jean-Luc; SIERPINSKA, Anna. Research into the teaching and learning of linear algebra. In: HOLTON, Derek et al. (Eds). The Teaching and Learning of Mathematics at University Level: an ICMI Study. Holanda: Kluwer Academic Publishers, 2001. p. 255-273. 
DUBINSKY, Ed. Reflective abstraction in advanced mathematical thinking. In: TALL, David. Advanced Mathematical Thinking, Holanda: Kluwer Academic Publishers, 2002, p. 95-123

DUBINSKY, Ed; MCDONALD, Michael A. APOS: A Constructivist Theory of Learning in Undergrad Mathematics Education Research. In D. Holton et al. (Eds.), The teaching and

Learning of Mathematics at University Level: An ICMI Study, Kluwer Academic Publishers, 2001, p. 273-280. Disponível em: <http://www.math.kent.edu/ edd/ICMIPaper.pdf>. Acesso em: 08 jul. 2014.

KARRER, Monica; JAHN, Ana Paula. Transformações lineares planas: um estudo com base nos registros de representação semiótica e na utilização da geometria dinámica. Boletim Gepem, Rio de Janeiro, n.55, p. 19-40, jul./dez., 2009. Disponível em:

<http://www.ufrrj.br/SEER/index.php?journal=gepem\&page=article\&op=view\&path\%5B\%5D=37 >. Acesso em: 01 jul. 2016.

KÚ, Darly; TRIGUEROS, Maria; OKTAÇ, Asuman. Comprensión del Concepto de base de un espacio vectorial desde el punto de vista de la teoria APOE. Educación Matemática, México, v. 20, n.2, 2008, p. 65-89. Disponível em: <http://www.redalyc.org/articulo.oa?id=40512062004>. Acesso em: 10 jul. 2014.

MORAES, Roque; GALIAZZI, Maria do C. Análise textual discursiva. Ijuí: Ed. Unijuí, 2007.

SOUZA, Mariany L. Dependência e independência linear: um estudo a respeito das dificuldades e concepções de licenciandos em Matemática. 2016. 126 f. Dissertação (Mestrado em Ensino de Ciências e Educação Matemática) - Universidade Estadual de Londrina, Londrina, 2016.

STEWART, Sepideh. Understanding linear algebra concepts through the embodied, symbolic and formal words of mathematical thinking. 2008. 300f. Tese (Doctor of philosophy of science in mathematics education) - University of Auckland, Auckland, 2008. Disponível em:

<https://researchspace.auckland.ac.nz/bitstream/handle/2292/2912/01front.pdf?sequence=1> Acesso em: 21 maio 2015.

TALL, David; VINNER, Shlomo. Concept image and concept definition in Mathematics wich particular reference to limits and continuity. Educational Studies in Mathematics, 12, p. 151-169, 1981.

Submetido em novembro de 2016 Aprovado em agosto de 2017 